\title{
Combined experimental and theoretical study on the differential elastic scattering cross sections for acetone by electron impact energy of 7.0-50 eV
}

\author{
D. F. Pastega,${ }^{1,2}$ E. Lange,${ }^{1}$ J. Ameixa, ${ }^{1}$ A. S. Barbosa,${ }^{1,2}$ F. Blanco, ${ }^{3}$ G. García,${ }^{4}$ M. H. F. Bettega, ${ }^{2}$ \\ P. Limão-Vieira, ${ }^{1,}{ }^{*}$ and F. Ferreira da Silva ${ }^{1}$ \\ ${ }^{1}$ Laboratório de Colisões Atómicas e Moleculares, CEFITEC, Departamento de Física, Universidade NOVA de Lisboa, \\ P-2829-516 Caparica, Portugal \\ ${ }^{2}$ Departamento de Física, Universidade Federal do Paraná, Caixa Postal 19044, Curitiba, Paraná 81531-990, Brazil \\ ${ }^{3}$ Departamento de Fisica Atomica, Molecular y Nuclear, Facultad de Ciencias Fisicas, Universidad Complutense de Madrid, \\ E-28040 Madrid, Spain \\ ${ }^{4}$ Instituto de Física Fundamental, Consejo Superior de Investigaciones Científicas (CSIC), Serrano 113-bis, E-28006 Madrid, Spain
}

(Received 28 January 2016; published 16 March 2016)

\begin{abstract}
We report elastic differential cross sections (DCSs) for electron interactions with acetone, $\mathrm{C}_{3} \mathrm{H}_{6} \mathrm{O}$. The incident electron energy range was 7.0-50 eV, and the scattered electron angular range for the differential measurements varied from $10^{\circ}$ to $120^{\circ}$. The calculated cross sections were obtained with two different methodologies, the Schwinger multichannel method with pseudopotentials (SMCPP), and the independent-atom method with screening-corrected additivity rule (IAM-SCAR). The present elastic DCSs have been found to agree well with the results of IAM-SCAR calculations above $20 \mathrm{eV}$, and also with the SMC calculations below $30 \mathrm{eV}$. Although some discrepancies were found for lower energies, the agreement between the SMCPP data and the DCSs obtained with the IAM-SCAR method improves, as expected, as the impact energy increases. Comparison with previous DCSs shows good agreement albeit the present data is extended down to lower electron impact energies. We find a low-lying $\pi^{*}$ shape resonance located at $2.6 \mathrm{eV}$, in agreement with recent results on electron collisions with acetone [M. G. P. Homem et al., Phys. Rev. A 92, 032711 (2015)]. The presence of a $\sigma^{*}$ resonance is also discussed.
\end{abstract}

DOI: 10.1103/PhysRevA.93.032708

\section{INTRODUCTION}

Acetone $\left(\mathrm{C}_{3} \mathrm{H}_{6} \mathrm{O}\right)$ has been extensively used in many industrial applications as a key agent in the manufacture of cordite and the strongest consumer-grade solvent. It is the simplest carbonyl [together with acetaldehyde $\left(\mathrm{CH}_{3} \mathrm{CHO}\right)$ ] and aliphatic compound that has been comprehensively studied as a model for the ketone family, with important investigations devoted to the electronic structure [1-7], its relevance within the interstellar medium [8], and even established as a biomarker for patients with diabetes [9-11]. Within DNA/RNA, hydrogen bonding is also performed between base pairs using the carbonyl groups which can be regarded as key structures in ketones, although, in the earlier stages of radiation-induced damage with the biological environment, low-energy electrons produced as the most abundant secondary species are relevant in driving much of the local radiation chemistry through certain initiation reactions [12]. Therefore, the study of electron interactions with simple ketones appears to be extremely relevant from a radiobiological point of view. Another key aspect of such studies is related to modeling the effects of low- and intermediate-energy electrons slowing down in biologically relevant materials (e.g., cross-section data and energy-loss distribution functions), where this information can be added as input parameters to be used to develop an effective dosimetric tool at the molecular level (i.e., nanodosimetry) [13,14].

The primary motivation for measurements of electronscattering cross-section data for $\mathrm{C}_{3} \mathrm{H}_{6} \mathrm{O}$ is the need for a

\footnotetext{
*Corresponding author: plimaovieira@ fct.unl.pt
}

reliable database of information that can be used to model such interactions and applications of this molecule.

Previous experimental work on this molecule includes the grand total cross-section (TCS) measurements by Szmytkowski [15] covering an energy range $0.7-400 \mathrm{eV}$, whereas Kimura et al. [16] covered the 0.8-600 eV range. $\mathrm{C}_{3} \mathrm{H}_{6} \mathrm{O}$ is a polar molecule with a large dipole moment (2.88 D) [17] and molecular polarizability $(\alpha)$ of considerable magnitude, $6.096 \AA^{3}$ [17], and we can anticipate that this is expected to play a key role in determining the magnitude and the angular and energy-dependent behavior of the low- to intermediate-energy electron-scattering cross sections. To our knowledge there is only one theoretical and experimental elastic differential cross section (DCS) for the $\mathrm{C}_{3} \mathrm{H}_{6} \mathrm{O}$ molecule by Homen et al. [18] at impact energies of 30-800 eV and $10^{\circ}-120^{\circ}$ scattering angles, however, there are no elastic DCSs for the $\mathrm{C}_{3} \mathrm{H}_{6} \mathrm{O}$ molecule in the low-energy range covered in the present work, i.e., below $30 \mathrm{eV}$.

The present study represents an experimental contribution to the measurement of elastic differential cross-section (DCS) data for $\mathrm{C}_{3} \mathrm{H}_{6} \mathrm{O}$, in particular, because it combines two complementary theoretical methodologies in assertion of the experimental data. Moreover, such an investigation allows one to present data in the intermediate- to low-energy range, i.e., below $30 \mathrm{eV}$, enabling us to present experimental and theoretical DCSs for electron impact energies as low as $7 \mathrm{eV}$.

In the next section we provide details on the experimental apparatus and the measurement techniques that have been used. In Sec. III we present a brief discussion on the Schwinger multichannel method (SMC) and the independentatom method with screening-corrected additivity rule 
(IAM-SCAR), and in Sec. IV the experimental results are presented, together with a discussion and comparison with other results, where that is possible. Finally, some concluding remarks that can be drawn from this study are given in Sec. V.

\section{APPARATUS AND OPERATING PROCEDURES}

The High Resolution Electron Energy Loss Spectrometer (HREELS) used in the Lisbon laboratory, VG-SEELS 400, has been described in detail elsewhere [19], so only a brief discussion will be presented here. A monochromatic electron beam is generated with a hemispherical electron monochromator and crossed at right angles with an effusive molecular beam that enters the interaction region through a hypodermic needle with a $0.95 \mathrm{~mm}$ inner diameter. After the electron interaction with the target gas, the scattered electrons are energy analyzed with a hemispherical electron analyzer, which can rotate about the gas jet, and can be detected by an electron multiplier. The typical base pressure in the main chamber was $5.0 \times 10^{-5} \mathrm{~Pa}$ and, upon gas admission $\left(\mathrm{C}_{3} \mathrm{H}_{6} \mathrm{O}\right)$, this increased to a pressure of $1.0 \times 10^{-3} \mathrm{~Pa}$. The liquid sample was supplied from Sigma Aldrich with a quoted purity of $>99.5 \%$. The sample was degassed by repeated freeze-pump-thaw cycles prior to use.

In the current experiments the energy resolution of the incident electron beam was $\sim 80 \mathrm{meV}$ [full width at half maximum (FWHM)], with incident electron currents of a few nA (depending on the initial electron energy). Such a value means that, in principle, there could be contributions to the elastic signal from some of the lower-lying vibrational modes of $\mathrm{C}_{3} \mathrm{H}_{6} \mathrm{O}$. However, in the energy range above $10 \mathrm{eV}$, these possible vibrational contributions are expected to be very small compared to the elastic intensity, and thus are not expected to make any significant contribution to the measured elastic cross sections, whereas below $10 \mathrm{eV}$, those have been extracted by deconvoluting the energy-loss spectra with the Gaussian profiles and separated from the elastic DCSs. The incident electron energy was calibrated to the elastic peak. The hemispherical electron analyzer is placed on a turntable stage that can be rotated from $0^{\circ}$ to $+120^{\circ}$, with respect to the incident electron beam, with an angular acceptance of $1.25^{\circ} \pm 0.25^{\circ}$

The absolute scale of the present differential cross sections (DCSs) was set by the theoretical results (see Sec. III) with the experimental data fitted and compared against the recent experimental results of Homem et al. [18] We estimate that the experimental uncertainties on the resulting $\mathrm{C}_{3} \mathrm{H}_{6} \mathrm{O}$ DCS lie in the range of $15 \%-20 \%$, with the actual value depending on the specific incident electron energy $\left(E_{0}\right)$ and scattered electron angle $(\theta)$ under consideration. This overall error largely consists of an uncertainty in keeping the correct flow conditions and, to a much lesser extent, an error associated with the statistical accuracy of the data and the stability of the incident electron beam $(<1 \%)$.

\section{COMPUTATIONAL METHODS}

To assist in the theoretical interpretation of the experimental DCSs, the present combined calculation methods permit one to study the interaction of low- to intermediate-energy $(\leqslant 30 \mathrm{eV})$ and intermediate- to high-energy $(\geqslant 20 \mathrm{eV})$ electrons with molecules. The former is based on the well-established Schwinger multichannel method (SMC) whereas the latter is based on a corrected form of the independent-atom model (IAM) known as SCAR (screening-corrected additivity rule). Details of these methods have been presented in previous works, therefore only a brief description is given here.

\section{A. Schwinger multichannel method (SMC)}

To compute the elastic cross sections of acetone (differential and integral), we employed the SMC method [20] with pseudopotentials (SMCPP) [21]. The SMC method and its implementations were recently reviewed in detail elsewhere [22]. Therefore, we will only discuss the theoretical aspects related to the present calculations. Our calculations were carried out at the ground-state geometry of acetone, which belongs to the $C_{2 v}$ symmetry group. The geometry of the molecular target was optimized at the second-order Møller-Plesset perturbation method (MP2) level and the TZV $++(2 d, 1 p)$ basis set, as implemented in the package GAMESS [23]. We used the pseudopotentials of Bachelet et al. [24] in order to replace the core electrons of the carbon and oxygen atoms, and the valence electrons are represented by five $s$-type, five $p$-type, and three $d$-type Cartesian Gaussian functions, generated according to Ref. [25]. For the hydrogens we employed the $4 s / 3 s$ basis set of Dunning [26] augmented with one $p$-type function with exponent 0.75 . The symmetric combinations of the $d$-type orbital were excluded to avoid linear dependency in the basis set.

The scattering cross sections were computed in the staticexchange plus polarization (SEP), where the configurationstate functions (CSFs) are built from products of target states with a single-particle wave function. In the simplest approximation, where only the static and exchange interactions are considered [known as the static-exchange (SE) approximation], the CSFs are given by a direct product between the target ground state, which is described in the Hartree-Fock level, and a single-particle function. In the SEP approximation, the direct space is augmented by considering direct products of $\mathrm{N}$-electron states, obtained by performing single (virtual) excitations of the target from the occupied (hole) orbitals to a set of unoccupied (particle) orbitals, and a single-particle function. We used the improved virtual orbitals (IVOs) [27] to represent the particle and scattering orbitals. In order to take the polarization effect into account, we considered single virtual excitations from all valence orbitals to all IVOs with energy below 1.0 hartree, resulting in the SEP calculation 12961 CSFs. Finally, we employed the standard Born-closure procedure [28] to account for scattering of the higher partial waves, due to the long-range character of the dipole potential. Our computed value for the dipole moment was $3.44 \mathrm{D}$, which is about $19 \%$ larger than the experimental value of $2.88 \mathrm{D}$ [17].

\section{B. Independent-atom model (IAM)}

Details of the application of the IAM-SCAR [29,30] method to electron interactions have been provided in a number of previous papers (see, e.g., Refs. [31-35]). Briefly, each atomic target $(\mathrm{C}, \mathrm{O}, \mathrm{H})$ is represented by an interacting complex 

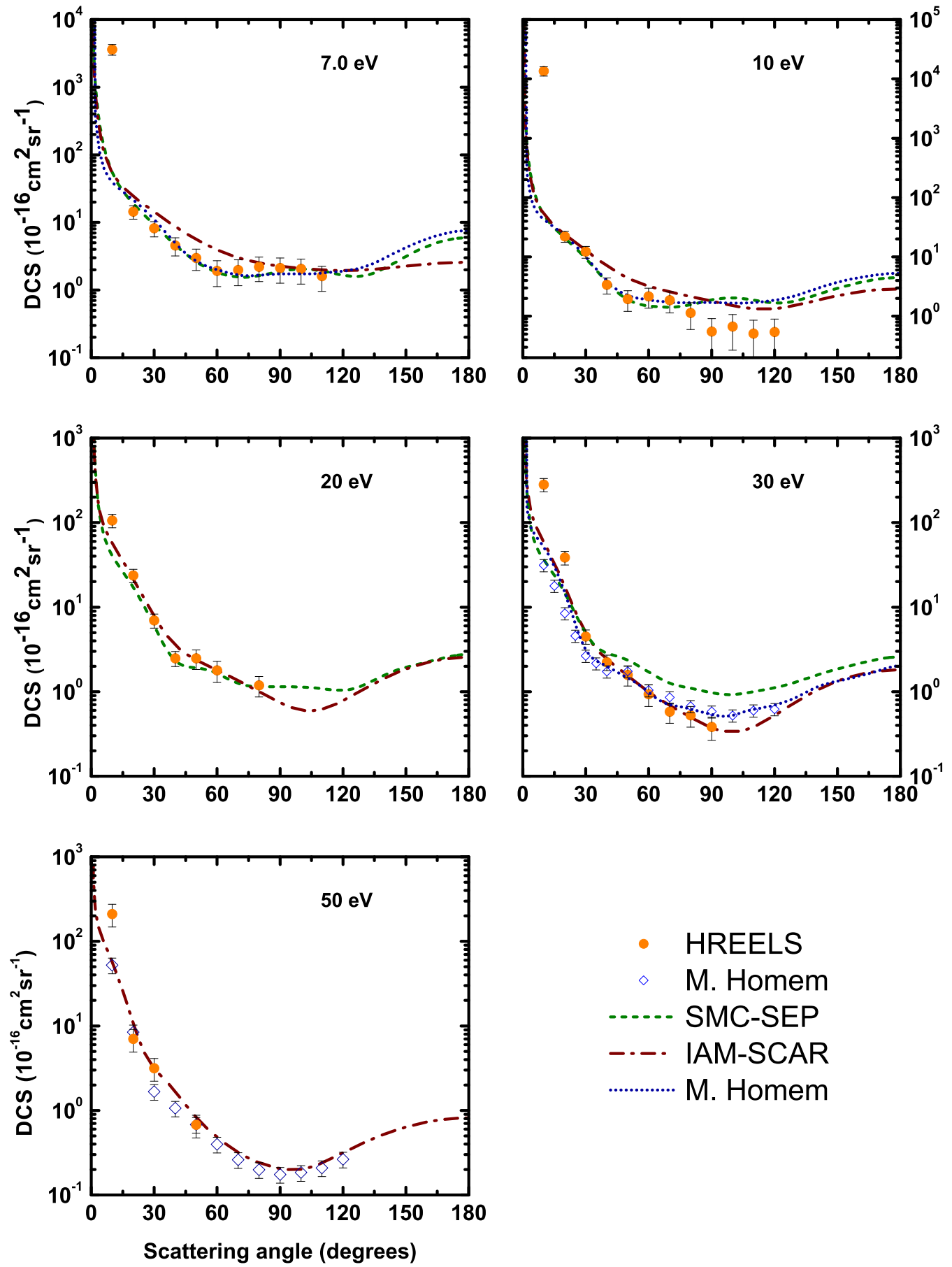

FIG. 1. Present DCS $\left(10^{-16} \mathrm{~cm}^{2} \mathrm{sr}^{-1}\right)$ for elastic electron scattering from $\mathrm{C}_{3} \mathrm{H}_{6} \mathrm{O}$ in the incident electron energy region 7.0-50 eV; $(\bullet)$ present elastic DCS; $(\diamond)$ Homen et al. [18] at 30 and $50 \mathrm{eV}$. Solid curves: Our SMC-SEP and IAM-SCAR calculations.

potential, the so-called optical potential. The real part accounts for the elastic scattering of the incident electrons, and the imaginary part represents the inelastic processes, which is considered to be "absorption" from the incident beam. For the elastic part, the potential is represented by the sum of three terms that include (a) a static term derived from a Hartree-Fock calculation of the atomic charge-density distribution, (b) an exchange term to account for the indistinguishability of the incident and target electrons, and (c) a polarization term for the long-range interactions which depends on the target polarizability. The inelastic scattering, on the other hand, is treated as electron-electron collisions. Further improvements to the original formulation in the description of the electron's indistinguishability and the inclusion of screening effects led to a model which provides a good approximation for electronatom scattering over a broad energy range. To calculate the cross sections for electron collisions with $\mathrm{C}_{3} \mathrm{H}_{6} \mathrm{O}$, the additivity rule (AR) is then applied to the optical model results for each constituent atom. In this approach, the molecular scattering amplitude stems from the coherent sum of all the 
relevant atomic amplitudes, which gives the DCSs for the molecule of interest. The geometry of the molecule (atomic positions and bond lengths) is taken into account by using some screening coefficients, and this enables the range of validity of the technique to be extended down to impact energies of $\sim 20 \mathrm{eV}$ for electron scattering.

\section{RESULTS AND DISCUSSION}

Figure 1 illustrates the angular distribution at five different electron impact energies, from 7.0 to $50 \mathrm{eV}$ for scattering angles from $10^{\circ}$ to $120^{\circ}$, together with the corresponding theoretical results from the application of the SMC-SEP and IAM-SCAR models and the previous experimental DCSs for acetone [18] at impact energies of 30 and $50 \mathrm{eV}$. Moreover, we include for comparison at 7, 10, and $30 \mathrm{eV}$ the DCS theoretical calculation by a molecular complex optical potential (MCOP) at the static-exchange polarization plus absorption (SEPA) level of approximation [18].

The absolute values of the cross sections are determined from the theoretical methods, from which the experimental data have been rescaled at a $30^{\circ}$ scattering angle, as well as from the recent experimental data of Homen et al. [18] to which we compare. All experimental DCSs are tabulated as numerical data in Table I. Here, we note that acetone cross sections are dominated by strong peaks in the forward-scattering direction. This is not surprising since the effects of the molecular dipole are most often seen at very small scattering angles. Notwithstanding, the large dipole polarizability also plays a key role in determining the magnitude and the angular and energy-dependent behavior of the low- to intermediateenergy electron-scattering cross sections. Note that the angular acceptance of the scattering apparatus $\left(<2^{\circ}\right)$ limits the ability of the experimental system to distinguish between unscattered and elastically or rotationally scattered electrons within the acceptance angle of the apparatus. Although the experimental data points at 7 and $10 \mathrm{eV}$ impact energy and $10^{\circ}$ scattering angle overestimate the theoretical data, the increasing trend of the DCS agrees with the expected behavior for the molecular properties of acetone (i.e., dipole moment and polarizability).

TABLE I. Experimental differential $\left(10^{-16} \mathrm{~cm}^{2} \mathrm{sr}^{-1}\right)$ cross sections for elastic scattering from $\mathrm{C}_{3} \mathrm{H}_{6} \mathrm{O}$. Errors on the DCSs are typically $15 \%-20 \%$.

\begin{tabular}{lrrrrr}
\hline \hline & \multicolumn{5}{c}{ Impact energy (eV) } \\
\cline { 2 - 6 } Angle (deg) & \multicolumn{1}{c}{7.0} & 10.0 & 20.0 & 30.0 & \multicolumn{1}{c}{50.0} \\
\hline 10 & 3615.75 & 13552.77 & 106.16 & 281.16 & 211.17 \\
20 & 14.42 & 22.17 & 23.69 & 38.56 & 6.99 \\
30 & 8.21 & 12.22 & 6.97 & 4.50 & 3.17 \\
40 & 4.57 & 3.83 & 2.48 & 2.23 & \\
50 & 2.98 & 1.95 & 1.92 & 1.57 & 0.68 \\
60 & 1.92 & 2.16 & 1.79 & 0.93 & \\
70 & 1.98 & 1.86 & & 0.57 & \\
80 & 2.20 & 1.14 & 1.19 & 0.52 & \\
90 & 2.12 & 0.55 & & 0.38 & \\
100 & 2.05 & 0.67 & & & \\
110 & 1.60 & 0.51 & & & \\
120 & \multicolumn{7}{c}{0.54} \\
\hline \hline
\end{tabular}

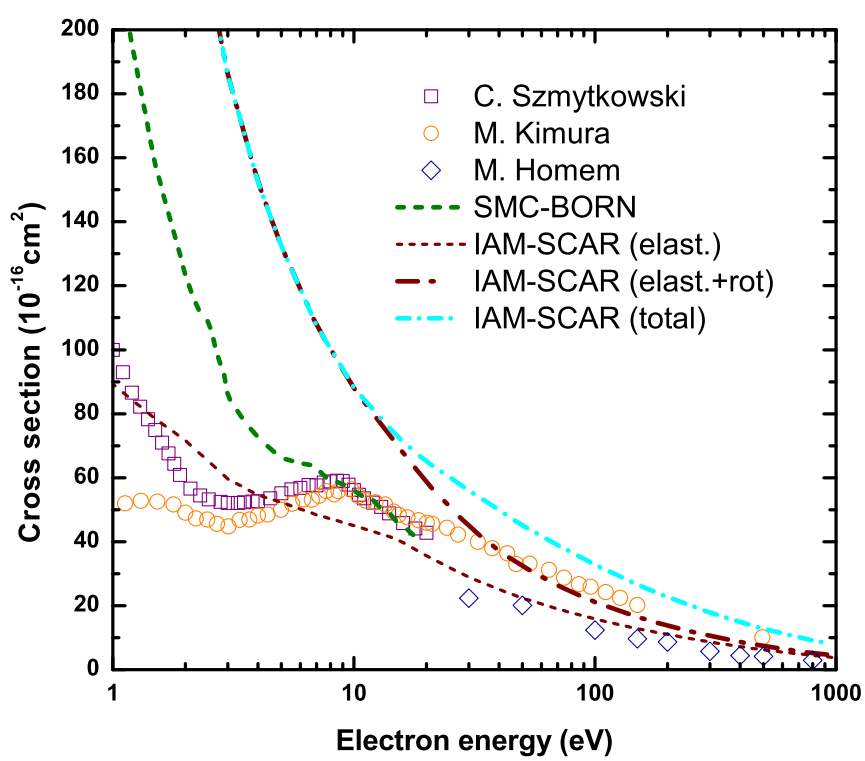

FIG. 2. Integral cross section for electron collisions with acetone. Our SMC-SEP results with Born-closure procedure, dashed line; total cross section of Szmytkowski [15], squares; total cross section of Kimura et al. [16], circles; integral cross section of Homem et al. [18], diamonds.

As shown in Fig. 1, the DCSs can be compared with the recent SMC-SEP and IAM-SCAR calculations. From the SMC-SEP method a very good agreement is observed up to $20 \mathrm{eV}$, whereas above this impact energy the theoretical DCS overestimates the experimental data. That is due to the approximation employed in the present calculations that considers only the elastic channel as an open channel and does not account for the inelastic processes that are already operational at those energies. Opening those inelastic channels would allow flux loss from the elastic channel to the inelastic ones, lowering the elastic cross sections towards the experimental data [36]. Regarding the IAM-SCAR method, a better agreement with the present experimental DCS in the electron energy range above $20 \mathrm{eV}$ is observed.

Below $20 \mathrm{eV}$, the DCSs for $\mathrm{C}_{3} \mathrm{H}_{6} \mathrm{O}$ show two discernible minima, one at $\sim 40^{\circ}$, which slightly shifts to larger scattering angles and becomes shallower at $7 \mathrm{eV}$, whereas the other is a less pronounced minimum close to $120^{\circ}-130^{\circ}$ at $7 \mathrm{eV}$, which progressively shifts to smaller scattering angles with increasing electron impact energies. Above $20 \mathrm{eV}$ electron impact, the first minimum shifts to lower scattering angles, becoming less pronounced, while the second minimum dominates and is clearly shifted to $90^{\circ}$ scattering at $50 \mathrm{eV}$. We are interested in the effect of the ${ }^{2} B_{1}$ and ${ }^{2} A_{2}$ resonances at $\sim 8 \mathrm{eV}$ [18] (see below) on the DCS, and the overall DCS behavior up to $50 \mathrm{eV}$. From Fig. 1 we can see that, generally speaking for all incident electron energies, the measured DCSs are peaked at the more forward-scattering angles, which is consistent with the significant magnitude $\left(6.096 \AA^{3}\right)$ of the dipole polarizability of $\mathrm{C}_{3} \mathrm{H}_{6} \mathrm{O}$.

Where comparable cross-section determinations exist from experiment and theory, our DCSs are in very good general agreement in overall shape with previous publications (see 


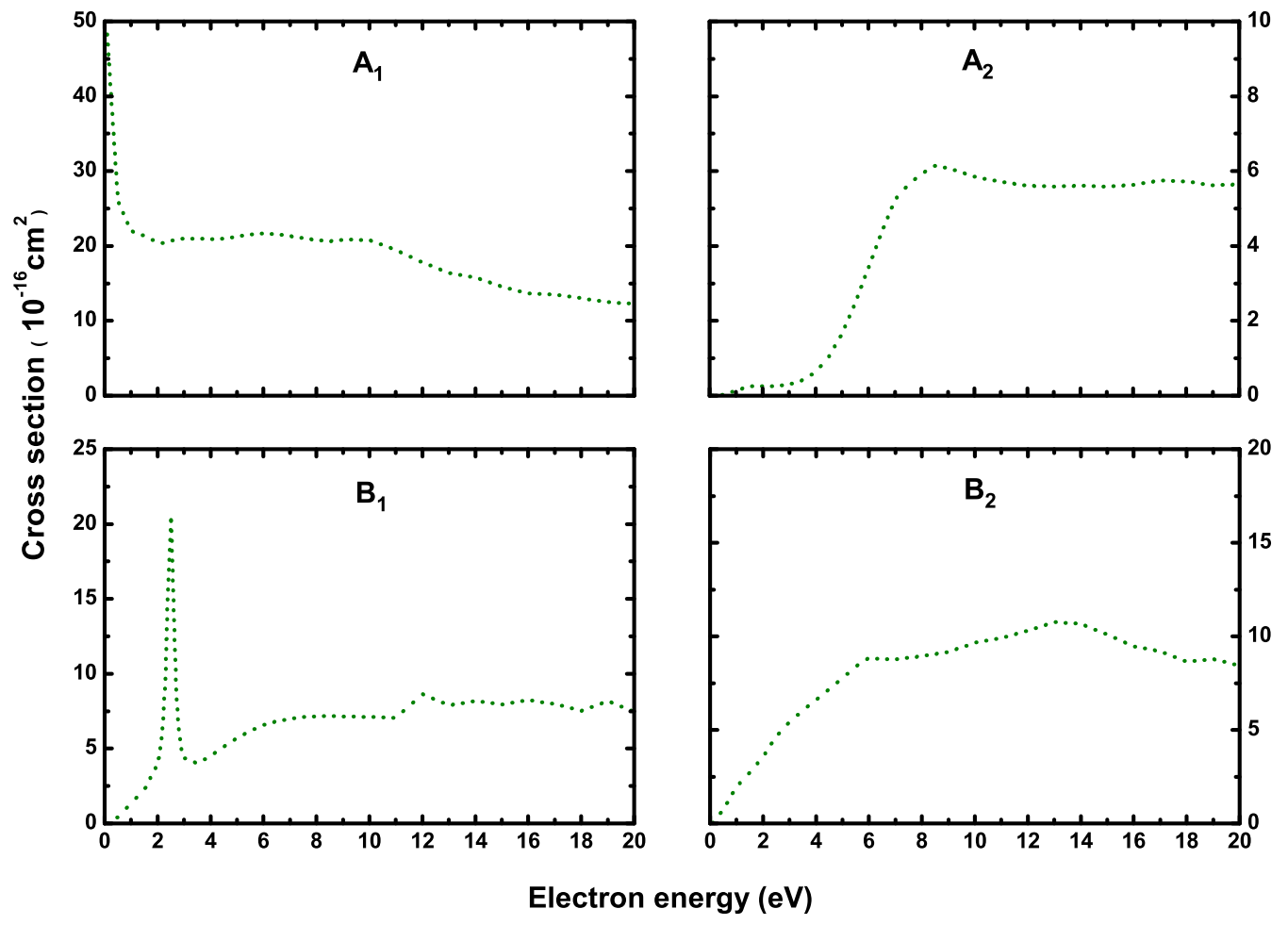

FIG. 3. Symmetry decomposition of the integral cross section for electron collision with acetone. See text for discussion.

Fig. 1 at 30 and $50 \mathrm{eV}$ and Fig. 5 in Ref. [18] at 10 and $20 \mathrm{eV}$, respectively). We may expect that several of the resonance features that are apparent in the total scattering measurements and theoretical calculations $[15,18]$ will also play a role in the nature of the DCS at energies below $10 \mathrm{eV}$.

The theoretical calculations of Homen et al. [18] reported that in the 8-20 eV collision energy they studied, the angular distributions show a rather oscillatory dependence on the scattering angle of the corresponding DCS being attributed to possible $d$-wave resonances. In general, the trends that they observe in the shape of the DCS at energies below about $20 \mathrm{eV}$ are also largely reflected in the present experimental results and are clearly discernible at 7 and $10 \mathrm{eV}$ electron impact energies. The broad feature at $\sim 8.5 \mathrm{eV}$ observed in the grand total cross section of Szmytkowski [15] has been attributed to resonant scattering through the formation of temporary negative-ion states. In such a scattering experiment [15], inelastic processes contributing to such a broad feature have also been proposed. In contention to this, Dorman [3] has reported several negativeion fragments in the vicinity of $9 \mathrm{eV}$. It is interesting to note other inelastic operative channels reported in the highresolution VUV electronic state spectroscopy of acetone [2], and the role of electronic excited valence and Rydberg states (with a vibronic structure) that are fully operational in this energy region. Finally, in the recent studies on the elastic electron scattering by acetone [18], such a broad feature was attributed to the weak ${ }^{2} B_{1}$ and ${ }^{2} A_{2}$ resonances and ${ }^{2} A_{1}$ shape resonance near $10 \mathrm{eV}$. This was proposed to be closely related to the double-dip behavior observed in the calculated DCS, which is observed here in Fig. 1 and is associated with these resonances. From these, the former may lead to dissociation mechanisms yielding the production of $\mathrm{H}$, $\mathrm{CH}_{2} \mathrm{CO}+\mathrm{CH}_{3}$, and $\mathrm{CO}+2 \mathrm{CH}_{3}$, whereas hydrogen atom abstraction occurs at $7.9 \mathrm{eV}$ following excitation of a $\left(\pi \rightarrow \pi^{*}\right)$ transition [37].

In their computed integral cross section, Homen et al. [18] also reported the presence of a $\pi^{*}$ shape resonance belonging to the ${ }^{2} B_{2}$ symmetry, and located at around $2.6 \mathrm{eV}$. This resonance is not observed in the TCS of Szmytkowski [15] and is barely seen in the TCS of Kimura et al. [16]. In Fig. 2 we show our elastic integral cross section (ICS) obtained in the SMC-SEP calculation, in comparison with the TCSs of Szmytkowski [15] and Kimura et al. [16], while in Fig. 3 we present the symmetry decomposition of the ICS. The SMC-SEP calculations also revealed this $\pi^{*}$ shape resonance located at around $2.4 \mathrm{eV}$. We also observed a $\sigma^{*}$ resonance in the ${ }^{2} A_{1}$ symmetry at around $8 \mathrm{eV}$, with major contributions coming from the partial waves with $l=2,3$, and 4 . It is worth mentioning that the low-lying $\pi^{*}$ shape resonance is masked by the Born closure in the SMC-SEP calculations. The long-range potential, along with nuclei vibration (that is not considered in our calculations), may be responsible for masking this resonance in the TCSs.

\section{CONCLUSIONS}

We report experimental elastic differential cross sections (DCSs) for electron scattering from acetone molecules, $\mathrm{C}_{3} \mathrm{H}_{6} \mathrm{O}$, together with a corresponding comparison to recent theoretical differential cross sections from the SMC-SEP with pseudopotentials and IAM-SCAR models. Agreement between these sets of data is generally very good in terms 
of the shapes and angular distributions of the cross sections. In addition, the corresponding IAM-SCAR formulations for electron energy above $20 \mathrm{eV}$ were found to be in good quantitative agreement with the present elastic DCS data, whereas below $20 \mathrm{eV}$, the experimental DCSs agree with the SMC-SEP calculations. The shapes of the angular distributions of the present DCSs, at each energy studied, are consistent with dipole effects playing a major role in governing the collision dynamics in this scattering system. We also found a $\pi^{*}$ shape resonance in the ${ }^{2} B_{2}$ symmetry at $2.6 \mathrm{eV}$, in agreement with the results of Homen et al. [18] that located the resonance at $2.4 \mathrm{eV}$. Finally, we report a broad $\sigma^{*}$ resonance in the ${ }^{2} A_{1}$ symmetry centered at around $8 \mathrm{eV}$, in accord with the experimental observations.

\section{ACKNOWLEDGMENTS}

We thank Professor M.-T. Lee from UFSCar, Brazil, for providing the theoretical data on DCS to be compared with the present results. D.F.P., A.S.B., and M.H.F.B. acknowledge the Brazilian Agency Coordenação de Aperfeiçoamento de Pessoal de Nível Superior (CAPES), under CAPES/FCT Programme (Process No. 23038.002465/2014-87). E.L. acknowledges the Brazilian Agency Conselho Nacional de Desenvolvimento Científico e Tecnológico (CNPq) and the Science Without Borders Programme for opportunities to study abroad. M.H.F.B. also acknowledges support from CNPq. F.F.S. acknowledges the Portuguese National Funding Agency FCT through Researcher Contract No. IF-FCT IF/00380/2014, and, together with P.L.V., Research Grants No. PTDC/FIS-ATO/1832/2012 and No. UID/FIS/00068/2013. This work has been partially supported by the Spanish MINECO (Project No. FIS2012-31230). We also acknowledge financial support from European Union programs (COST CM1301 and FP7-ITN ARGENT). This work was also supported by FCT-MCTES, Radiation Biology and Biophysics Doctoral Training Programme (RaBBiT, PD/00193/2012); UID/Multi/04378/2013 (UCIBIO).
[1] P. Limão-Vieira, S. Eden, N. J. Mason, and S. V. Hoffmann, Chem. Phys. Lett. 376, 737 (2003).

[2] M. Nobre, A. Fernandes, F. Ferreira da Silva, R. Antunes, D. Almeida, V. Kokhan, S. V. Hoffmann, N. J. Mason, S. Eden, and P. Limão-Vieira, Phys. Chem. Chem. Phys. 10, 550 (2008).

[3] F. H. Dorman, J. Chem. Phys. 44, 3856 (1966).

[4] W. T. Naff, R. N. Compton, and C. D. Cooper, J. Chem. Phys. 57, 1303 (1972).

[5] M. V. Muftakhov and A. I. Fokin, Rapid Commun. Mass Spectrom. 11, 1923 (1997).

[6] E. Illenberger and M. C. Meinke, Int. J. Mass Spectrom. 365366, 80 (2014).

[7] V. S. Prabhudesai, V. Tadsare, S. Ghosh, K. Gope, D. Davis, and E. Krishnakumar, J. Chem. Phys. 141, 164320 (2014).

[8] L. E. Snyder, F. J. Lovas, D. M. Mehringer, N. Y. Miao, Y.-J. Kuan, J. M. Hollis, and P. R. Jewell, Astrophys. J. 578, 245 (2002).

[9] A. Manolis, Clin. Chem. 29, 5 (1983).

[10] K. Musa-Veloso, E. Rarama, F. Comeau, F. Curtis, and S. Cunnane, Pediatr. Res. 52, 443 (2002).

[11] F. Ferreira da Silva, M. Nobre, A. Fernandes, R. Antunes, D. Almeida, G. García, N. J. Mason, and P. Limão-Vieira, J. Phys.: Conf. Ser. 101, 012011 (2008).

[12] B. Boudaïffa, P. Cloutier, D. Hunting, M. A. Huels, and L. Sanche, Science 287, 1658 (2000).

[13] F. Blanco, A. Muñoz, D. Almeida, F. Ferreira da Silva, P. LimãoVieira, M. J. Brunger, S. J. Buckman, M. C. Fuss, A. G. Sanz, and G. García, Eur. Phys. J. D 67, 199 (2013).

[14] A. G. Sanz, M. C. Fuss, A. Muñoz, F. Blanco, P. Limão-Vieira, M. J. Brunger, S. J. Buckman, and G. García, Int. J. Radiat. Biol. 88, 71 (2012).

[15] C. Szmytkowski, J. Phys. B: At. Mol. Opt. Phys. 43, 055201 (2010), and references therein.

[16] M. Kimura, O. Sueoka, A. Hamada, and Y. Itikawa, Adv. Chem. Phys. 111, 537 (2000).

[17] http://cccbdb.nist.gov/.
[18] M. G. P. Homem, I. Iga, L. A. da Silva, J. R. Ferraz, L. E. Machado, G. L. C. de Souza, V. A. S. da Mata, L. M. Brescansin, R. R. Lucchese, and M.-T. Lee, Phys. Rev. A 92, 032711 (2015).

[19] F. Motte-Tollet, M.-J. Hubin-Franskin, and J. E. Collin, J. Chem. Phys. 97, 7314 (1992).

[20] K. Takatsuka and V. McKoy, Phys. Rev. A 24, 2473 (1981); 30, 1734 (1984).

[21] M. H. F. Bettega, L. G. Ferreira, and M. A. P. Lima, Phys. Rev. A 47, 1111 (1993).

[22] R. F. da Costa, M. T. do N. Varella, M. H. F. Bettega, and M. A. P. Lima, Eur. Phys. J. D 69, 159 (2015).

[23] M.W. Schmidt, K. K. Baldridge, J. A. Boatz, S. T. Elbert, M. S. Gordon, J. H. Jensen, S. Koseki, N. Matsunaga, K. A. Nguyen, S. J. Su, T. L. Windus, M. Dupuis, and J. A. Montgomery, J. Comput. Chem. 14, 1347 (1993).

[24] G. B. Bachelet, D. R. Hamann, and M. Schlüter, Phys. Rev. B 26, 4199 (1982).

[25] M. H. F. Bettega, A. P. P. Natalense, M. A. P. Lima, and L. G. Ferreira, Int. J. Quantum Chem. 60, 821 (1996).

[26] T. H. Dunning, Jr., J. Chem. Phys. 53, 2823 (1970).

[27] W. J. Hunt and W. A. Goddard III, Chem. Phys. Lett. 3, 414 (1969).

[28] E. M. de Oliveira, M. T. do N. Varella, M. H. F. Bettega, and M. A. P. Lima, Eur. Phys. J. D 68, 65 (2014).

[29] F. Blanco and G. García, Phys. Lett. 360, 707 (2007).

[30] F. Blanco, J. Rosado, A. Illana, and G. García, Phys. Lett. A 374, 4420 (2010).

[31] P. Limão-Vieira, M. Horie, H. Kato, M. Hoshino, F. Blanco, G. García, S. J. Buckman, and H. Tanaka, J. Chem. Phys. 135, 234309 (2011).

[32] H. Kato, A. Suga, M. Hoshino, F. Blanco, G. García, P. Limão-Vieira, M. J. Brunger, and H. Tanaka, J. Chem. Phys. 136, 134313 (2012).

[33] H. Kato, K. Anzai, T. Ishihara, M. Hoshino, F. Blanco, G. García, P. Limão-Vieira, M. J. Brunger, S. J. Buckman, and H. Tanaka, J. Phys. B: At. Mol. Opt. Phys. 45, 095204 (2012). 
[34] H. Murai, Y. Ishijima, T. Mitsumura, Y. Sakamoto, H. Kato, M. Hoshino, F. Blanco, G. García, P. Limão-Vieira, M. J. Brunger, S. J. Buckman, and H. Tanaka, J. Chem. Phys. 138, 054302 (2013).

[35] M. Hoshino, P. Limão-Vieira, K. Anzai, H. Kato, H. Cho, D. Mogi, T. Tanioka, F. Ferreira da Silva, D. Almeida, F. Blanco, G. García, O. Ingólfsson, and H. Tanaka, J. Chem. Phys. 141, 124302 (2014).
[36] See, for example, R. F. da Costa, M. H. F. Bettega, M. T. do N. Varella, E. M. de Oliveira, and M. A. P. Lima, Phys. Rev. A 90, 052707 (2014); R. F. da Costa, E. M. de Oliveira, M. H. F. Bettega, M. T. do N. Varella, D. B. Jones, M. Brunger, F. Blanco, R. Colmenares, P. Limão-Vieira, G. García, and M. A. P. Lima, J. Chem. Phys. 142, 104304 (2015), and the references therein.

[37] R. H. Huebner, R. J. Cellota, S. R. Mielczarek, and C. E. Kuyatt, J. Chem. Phys. 59, 5434 (1973). 